\title{
Anomalous Branches of Median Nerve In The Arm
}

\author{
Sunita Bharati ${ }^{1}$, N. Sujatha ${ }^{2}$, S. Swayam Jothi ${ }^{3}$ \\ ${ }^{1 .}$ Assistant Professor, Dept of Anatomy, Shri Sathya Sai Medical College \& Research Institute Ammapettai- \\ 603108, Chennai (T.N), India \\ ${ }^{2}$ Tutor, Dept of Anatomy, Shri Sathya Sai Medical College \& Research Institute Ammapettai-603108, Chennai \\ (T.N), India \\ ${ }^{3}$.Professor \& Head, Dept of Anatomy, Shri Sathya Sai Medical College \& Research Institute Ammapettai- \\ 603108, Chennai (T.N), India
}

\begin{abstract}
Variations of the median nerve at the level of brachial plexus are common. During routine dissection we observed branches of median nerve in the arm which supplies muscles of arm in a sixty five year old male cadaver right upper limb. Musculocutaneous nerve was absent and branch from median nerve supply corachobrachialis. And other branch from median nerve pierces biceps brachii and brachialis muscle by separate branches and continue as lateral cutaneous nerve of forearm.
\end{abstract}

Keywords: Absence of musculocutaneous nerve, Variation of median nerve,

\section{Introduction}

Anomalies of the brachial plexus and its terminal branches are not uncommon. Brachial plexus is network of nerves supplying the upper limb. Median nerve is formed in axilla by union of lateral root (C5-7) and medial root $(\mathrm{C} 8, \mathrm{~T} 1)$ of lateral \& medial cord of brachial plexus respectively [1]. Variations in the formation and branching of brachial plexus are reported by several investigators. Median nerve doesn't give any branch to muscles of front of arm which are supplied by musculocutaneous nerve only.

The aim of this case report is to highlight unilateral (right) absence of musculocutaneous nerve, and branches from median nerve after supplying all the muscles of front of arm, one branch continued as lateral cutaneous nerve of forearm.

The knowledge of the anatomical variations of the peripheral nerves in the upper limbs is important as these abnormal nerves could be injured during surgical procedures [2]

Abbrevations: $\mathrm{MN}$ - median nerve, $\mathrm{MCN}$-musculocutaneous nerve, $\mathrm{CBR}$ - corachobrachialis, BB- biceps brachii, BR-brachialis, LCNF - lateral cutaneous nerve of forearm

\section{Case Report}

65 year-old male cadaver for 1st year MBBS students, the following unusual findings were encountered in the Department of Anatomy, Sri Sathya Sai Medical College, Ammapetai., Chennai. A longitudinal incision was made at the anterior aspect of the arm, from the level of acromion process to a point about $2.5 \mathrm{~cm}$ below the elbow joint. A horizontal incision was made bilaterally in both proximal and distal ends of the longitudinal incision. The subcutaneous fat, fascia and muscles of arm were separated to observe the branches of lateral cord and additional branches from median nerve.

In right axilla, cords of brachial plexus were exposed after retracting pectoral muscles. MCN was found to be completely absent. The nerve to coracobrachialis was arising as a small branch directly from lateral root of median nerve. After that, the lateral root and medial root coming from either side of axillary artery joined to form $\mathrm{MN}$ proper in front of the artery.

In the middle of arm, two branches were seen coming from lateral side of MN. One branch (proximal) splits into two and supplied short and long heads of biceps brachii; the other distal branch splits into four branches, out of four proximal three pierces brachialis and distal branch by crossing biceps tendon continues lateral cutaneous nerve of forearm supplying lateral side of skin of forearm. On further dissection, the distribution of median nerve was found to be normal in forearm and palm. No such variation was detected on left side.

\section{III.Discussion}

Median nerve as reported in literature, is associated with several variations which include abnormal communications with other nerves such as musculocutaneous and ulnar nerves [4], splitting of the median nerve [5] and unusual innervations of flexor muscles of arm by the median nerve[6]. 
In present study, musculocutaneous nerve was absent and all muscles of front of arm are supplied by median nerve. According to Le Minor's description [3], there are five types of variations: Type 1: No communication between MN and MCN; Type 2: The fibers of the medial root of the MN pass through the MCN and join the MN in the middle of the arm; Type 3: The fibers of the lateral root of the MN pass through the MCN and after some distance leave it to form the lateral root of the MN; Type 4: The MCN fibers join the lateral root of the MN and after some distance the MCN arise from the MN; Type 5: The MCN is absent and all the MCN fibers pass through lateral root of MN; fibers to the muscles supplied by MCN branch out directly from MN. In this type, the MCN does not pierce the coracobrachialis muscle. In present study, lateral root of MN substituted MCN indicating type 5 of Le Minor's variant.

\section{Conclusion}

The anomalous branches of median nerve in the arm bear remarkable clinical significance. Some authours suggested that the clinicians and surgeons should be cautious of such variations while performing surgical procedure in this region [7]. When Injury occurs to such a variant nerve in the proximal arm, there may be sensory motor, vasomotor and trophic changes in the distal area to the injury [8]. These variations have also clinical importance when a surgeon is approaching for operating on an entrapment syndromes in the arm [9]. Hence this study will enrich the knowledge of surgeons about the possible anomalous branches of median nerve in the arm and so that they will remain cautious while operating on that area.

\section{References}

[1]. Gray's Anatomy, 40 ${ }^{\text {th }}$ edition. Edinburgh, London, Newyork, Philadelphia, Sydeny, Toronto: Churchill Livingstone, 2008: 781,790, $821,822$.

[2]. Bergmann RA, Thomson SA, Saadeh FA. Compendium of human anatomic variation Urban \& Schwarzebergmunich.1988; 139143.

[3]. Le Minor JM. A rare variation of the median and musculocutaneous nerves in man. Arch Anat .Histol. Embryol. 1990; 73 : 33-42. (French)

[4]. Chauhan, R, Roy TS. Communication between the median and musculocutaneou nerve- A case report. Journal of anatomical society of India 2002; $51: 72-5$

[5]. Sundaram, SM, Kumar MSJ, Sethupathi BB, Nayak S, Krishnamurthy A. Split median nerve with variation in its common digital branch. Neuroanatomy 2008; 7: 15-6.

[6]. Nayak S. Absence of musculocutaneous nerve associated with clinically important variations in the formation, course and distribution of the median nerve. Neuroanatomy 2007; 6; 49-50

[7]. Rao PPV, Chaudhary SC. Communication of musculocutaneous nerve with the median nerve. East Afr Med J 2000; 77: 498-503

[8]. Saeed M, Rufai AA. Median and musculocutaneous nerves: Variant formation and distribution. Clin Anat 2003; 16: 453-7.

[9]. Venieratos D, Anagnostopoulou S. Classification of communication between musculocutaneous and median nerves. Clin Anat 1998; 11: 327-31.

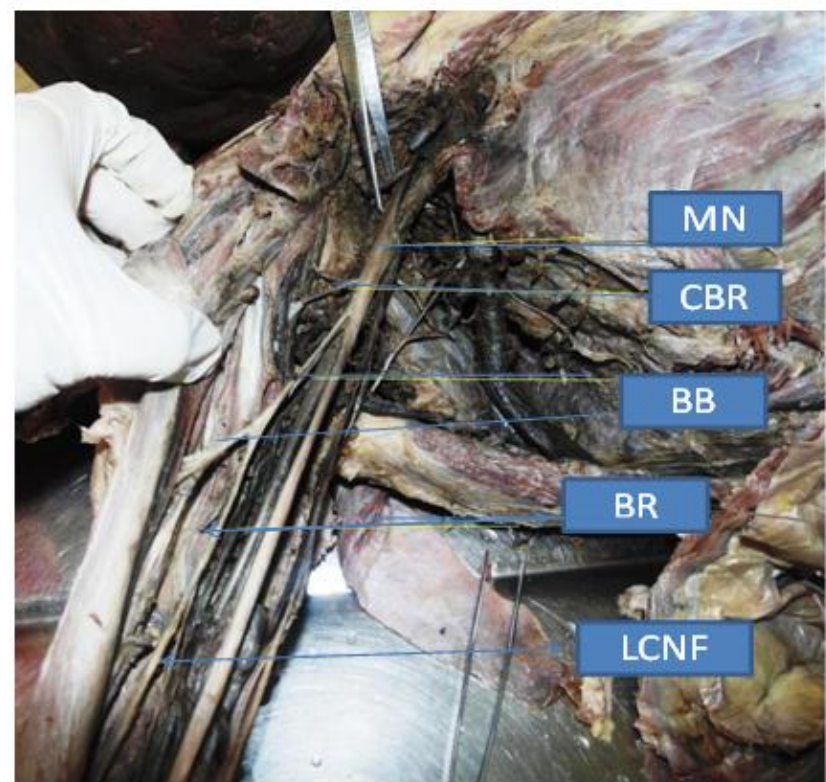

Photograph of right axilla of front of arm showing absence of musculocutaneous nerve and variant branches of median nerve (MN) supplying coracobrachialis (CBR), biceps brachii (BB) and brachialis (BR) muscles and continued as lateral cutaneous nerve of the forearm (LCNF) 